\title{
A disequilibrium reactive transport model for mantle magmatism
}

\author{
B. OLIVEIRA ${ }^{1 *}$, J.C. AFONSO ${ }^{1,2}$, M. KLÖCKING ${ }^{3}$, \\ R. TILHAC ${ }^{1,4}$
}

${ }^{1}$ ARC Centre of Excellence for Core to Crust Fluid Systems

(CCFS) and GEMOC, Department of Earth and Planetary Sciences, Macquarie University, Australia

${ }^{2}$ Centre for Earth Evolution and Dynamics, University of Oslo, Norway

${ }^{3}$ Research School of Earth Sciences, Australian National University, Australia

${ }^{4}$ Faculty of Geosciences and Civil Engineering, Institute of Science and Engineering, Kanazawa University, Japan (*correspondence: oliveira.bravo.b@gmail.com)

Besides standard thermo-mechanical considerations, a general description of mantle magmatism requires the simultaneous consideration of phase changes (e.g. from solid to liquid), chemical reactions (i.e. exchange of chemical components) and multiple dynamic phases (e.g. liquid percolating through a deforming matrix). Typically, these processes evolve at different rates, over multiple spatial scales and exhibit complex feedback loops and disequilibrium features.

Here we present a conceptual and numerical model that provides a versatile platform to study the dynamics and nonlinear feedbacks inherent to mantle magmatism and to make quantitative comparisons between petrological and geochemical datasets. Our model is based on the combination of three main modules: i) a Two-Phase, Multi-Component, Reactive Transport module that describes how liquids and solids evolve in space and time [1], ii) a melting formalism, called Dynamic Disequilibirum Melting, based on thermodynamic grounds and capable of describing disequilibrium major-element chemical exchange between phases, and iii) a grain-scale model for diffusion-controlled trace-element mass-transfer. We apply this integrated approach to decompression melting and show how the composition and volumes of melting products (e.g. MORBs) are consequence of their specific thermal, chemical and mechanical history. Finally, we use inverse modelling methods to further constraint used model parameters, provide better match between model results and field observations, and estimate uncertainties. This joint geochemical-geophysical inverse approach has broad applicability, and is readily extendable to accommodate additional processes of geochemical relevance, including refertilization processes, melting with volatiles, and/or melting of heterogeneous sources, among others. 